\title{
Docetaxel in der First- und Second-line-Therapie des Magenkarzinoms
}

\author{
Docetaxel in the First- and Second-LineTherapy of Gastric Cancer
}

\author{
U. Vanhoefer \\ Innere Klinik und Poliklinik (Tumorforschung), Westdeutsches Tumorzentrum, Universitätsklinikum Essen
}

D ie Prognose bei Patienten mit fortgeschrittenem M agenkarzinom ist als äußerst schlecht einzustufen. Eine A nalyse an 1700 Patienten zeigte, dass die 5-Jahres-Ü berlebensrate bei Patienten mit lokal fortgeschrittenen Tumoren im Stadium III bei etwa $20 \%$ bzw. im Stadium IV mit resektablen Tumoren bei 15\% liegt [1]. Patienten mit inoperablen Tumoren haben unbehandelt lediglich eine mediane Lebenserwartung von knapp $3 \mathrm{M}$ onaten. In randomisierten Studien konnte gezeigt werden, dass eine auf 5-Fluorouracil basierende Chemotherapie sowohl die Überlebenszeit als auch die Lebensqualität von Patienten mit fortgeschrittenem Magenkarzinom signifikant verbessert. Im Laufe der letzten Jahre wurden zahlreiche solcher 5-Fluorouracil-basierten Chemotherapieprotokolle konzipiert, die zumeist ein A nthrazyklin und/oder Cisplatin enthielten. Die A nsprechraten dieser Chemotherapieprotokolle lagen in Phase-II-Studien im Bereich von 30-50\%, die mediane Ü berlebenszeit bei etwa 8-11 M onaten. In randomisierten Studien wurden diese E rgebnisse aber zumeist nicht erreicht, so auch nicht in der EORTC-Studie 40902, in der das E LF-R egime (E toposid, Folinsäure, 5-Fluorouracil) sowie das 5-Fluorouracil/Cisplatin-R egime mit FA M TX (5-Fluorouracil, $D$ oxorubicin, $M$ ethotrexat) verglichen wurde. In dieser Studie betrug die $\mathrm{R}$ ate an objektiven R emissionen unter $20 \%$ für die drei verschiedenen Therapiearme [2]. In einer randomisierten Studie, in der ECF (E pirubicin, Cisplatin, 5-Fluorouracil) mit dem FAMTX-Regime verglichen wurde, zeigte sich eine signifikante Ü berlegenheit zugunsten des ECF-R egimes (A nsprechrate 45 vs. 21\%, mediane Überlebenszeit 8,9 vs. 5,7 M onate) [3]. A llerdings betrug in dieser Studie der A nteil von Patienten mit lokal fortgeschrittenen Tumoren 30\%, und ein Teil dieser Patienten wurde sekundär operiert. D ennoch betrug die Ü berlebensrate nach 2 Jahren auch im ECF-A rm lediglich noch $11 \%$. In einer extendierten Phase-II-Studie mit einer wöchentlichen Chemotherapie mit Folinsäure und 5-Fluorouracil (modifiziertes A IO-Schedule) in Kombination mit 2wöchentlicher Cisplatin-G abe lag die A nsprechrate bei etwa $60 \%$ für Patienten mit metastasierter Erkrankung mit einer medianen Ü berlebenszeit von 11 Monaten [4]. Von Bedeutung ist, dass mit dem Infusionsregime aus 5-Fluorouracil und Cisplatin Patienten mit einer Peritonealkarzinose keine wesentlich schlechtere Prognose hatten. Basierend auf diesen
D aten sollte die Chemotherapie mit 5-Fluorouracil (Infusionsschema) in Kombination mit Cisplatin als aktuelles Standardregime beim fortgeschrittenen $M$ agenkarzinom angegeben werden, an denen neue Therapieprotokolle zu messen sind. $\mathrm{E}$ in $\mathrm{N}$ achteil der I nfusionsregime ist allerdings die N otwendigkeit der Implantation eines $\mathrm{H}$ ickman-K atheters bzw. eines Portsystems, welches zum Teil für den Patienten belastend bzw. mit Komplikationen behaftet sein kann. A n neuen Substanzen, die zukünftig eine R olle in der Therapie des M agenkarzinoms spielen könnten, stehen vor allem Vertreter von 4 Wirkstoffgruppen zur Verfügung: Topoisomerase-I-H emmer wie I rinotecan, Taxane (D ocetaxel, Paclitaxel), orale Prodrugs von 5-Fluorouracil (U FT, Capecitabine) sowie neue Platinverbindungen wie $O$ xaliplatin.

\section{First-line-Therapie mit Docetaxel}

D ocetaxel wurde in 4 Phase-II-Studien in Europa, Japan und Nordamerika in der First-line-Therapie des fortgeschrittenen $M$ agenkarzinoms geprüft [5-8]. In 3 dieser Studien wurde Docetaxel in einer Dosierung von $100 \mathrm{mg} / \mathrm{m}^{2}$ alle 3 Wochen eingesetzt [5-7], in der japanischen Studie in der niedrigeren D osierung von $60 \mathrm{mg} / \mathrm{m}^{2}$ alle 3-4 Wochen. D ennoch wurde in den Studien eine einheitliche Remissionsrate von $17-24 \%$ erzielt. Die mediane Ü berlebenszeit wurde in 2 Studien angegeben und lag dort bei 7,2 bzw. 7,9 M onaten (Tab. 1). In der Z usammenfassung der 4 Studien mit insgesamt 159 Patienten ergibt sich eine A nsprechrate von 21\% (95\% -K onfidenzintervall $15-28 \%$ ) und eine Tumorwachstumskontrollrate von $51 \%$ (95\% -K onfidenzintervall 43-59\%).

$\mathrm{H}$ insichtlich der N ebenwirkungen stand die $M$ yelosuppression im Vordergrund. Eine Neutropenie G rad 3/4 trat bei mehr als $80 \%$ der Patienten auf. D ie H äufigkeit anderer N ebenwirkungen wie A bgeschlagenheit, Ü belkeit/E rbrechen, periphere Ödeme oder Überempfindlichkeitsreaktionen lag unter 10-15\%. Von klinischer Bedeutung ist, dass die hämatologische Toxizität von Docetaxel im Laufe der Therapie nicht kumuliert. In der Studie von Sulkes et al. [5], in der $37 \mathrm{~Pa}$ tienten insgesamt 156 Zyklen Docetaxel erhielten, betrug der mediane Granulozytennadir im ersten Zyklus 0,47

\begin{tabular}{ll}
\hline KARGER & ○ 2000 S. Karger G mbH , Freiburg \\
Fax +49 761 4520714 & A ccessible online at: \\
$\begin{array}{l}\text { E-mail Information@K arger.de } \\
\text { www.karger.com }\end{array}$ & www.karger.com/journals/onk
\end{tabular}

Dr.U.Vanhoefer

U niversitätsklinikum E ssen

Innere K linik und Poliklinik (Tumorforschung)

$\mathrm{H}$ ufelandstraße 55

D -45147 E ssen (G ermany) 
Tab. 1 Phase-II -M onotherapiestudien mit D ocetaxel bei unvorbehandelten Patienten mit fortgeschrittenem M agenkarzinom (mod. nach Vanhoefer)

\begin{tabular}{|c|c|c|c|c|c|c|c|}
\hline \multirow[t]{2}{*}{ Autor } & \multirow{2}{*}{$\begin{array}{l}\text { Patienten } \\
\mathrm{n}\end{array}$} & \multirow{2}{*}{$\begin{array}{l}\text { Dosis } \\
\mathrm{mg} / \mathrm{m}^{2}\end{array}$} & \multirow[t]{2}{*}{ Schema } & \multicolumn{3}{|c|}{ komplette + partielle R emission } & \multirow{2}{*}{$\begin{array}{l}\text { M edianes Ü berleben, } \\
\text { M onate }\end{array}$} \\
\hline & & & & $\bar{n}$ & $\%$ & $95 \%-\mathrm{KI}, \%$ & \\
\hline Sulkes et al., 1994 [5] & 37 & 100 & alle 3 Wochen & 8 & 22 & $8-35$ & - \\
\hline A ndroulakis et al., 1996 [6] & 22 & 100 & alle 3 Wochen & 5 & 23 & $5-41$ & 7,2 \\
\hline E inzig et al., 1996 [7] & 41 & 100 & alle 3 Wochen & 7 & 17 & $5-29$ & 7,9 \\
\hline Taguchi et al., 1998 [8] & 59 & 60 & alle 3-4 Wochen & 14 & 24 & $14-37$ & - \\
\hline
\end{tabular}

(Bereich $0,04-3,2) \times 10^{9} / 1$ und im letzten Zyklus 0,71 (Bereich $0,04-2,3) \times 10^{9} /$. D er durchschnittliche Thrombozytennadir lag im 1. Zyklus bei 271 (Bereich: $205-474) \times 10^{9} / l$ und im letzten Zyklus bei 278 (Bereich: $215-613) \times 10^{9} /$. Auch therapiebedingte $A$ nämien traten nicht auf.

In 3 Phase-II-Studien wurde Docetaxel in der First-lineTherapie mit anderen Zytostatika kombiniert [9-11]. In den beiden Studien mit der Kombination Docetaxel/Cisplatin wurde Cisplatin jeweils in der D osierung $75 \mathrm{mg} / \mathrm{m}^{2}$ und D ocetaxel in der Dosierung von 75 [9] bzw. $85 \mathrm{mg} / \mathrm{m}^{2}$ [10] verabreicht, die Therapie wurde alle 3 Wochen wiederholt. Die A nsprechraten bei 43 bzw. 53 auswertbaren Patienten betrugen 39,5 bzw. $45 \%$, die mediane Ü berlebenszeit wurde mit 13,1 bzw. 8,6 M onaten angegeben. In einer weiteren Studie wurde Docetaxel $75-100 \mathrm{mg} / \mathrm{m}^{2}$ mit wöchentlichen Dosen von Folinsäure/5-Fluorouracil $\left(500 / 1800 \mathrm{mg} / \mathrm{m}^{2}\right)$ kombiniert [11]. D ie A nsprechrate dieser Therapie lag bei 22\% (4/18 Patienten), die mediane Ü berlebensdauer betrug $8,0 \mathrm{M}$ onate. R oth et al. [12] setzten in einer Phase-I-Studie bei 39 Patienten die D reierkombination Docetaxel, Cisplatin und 5-Fluorouracil ein. A Is empfohlene D osis für weitere Phase-II-Studien ermittelten sie D ocetaxel $85 \mathrm{mg} / \mathrm{m}^{2}$, Tag 1 , Cisplatin $75 \mathrm{mg} / \mathrm{m}^{2}$, Tag 1 , und 5-F luorouracil $300 \mathrm{mg} / \mathrm{m}^{2} / T a g$ als $D$ auerinfusion, Tag 1- 14, mit Zykluswiederholung alle 3 Wochen. D aten zur Wirksamkeit dieser Kombination liegen noch nicht vor.

\section{Second-line-Therapie mit Docetaxel}

In einer in Deutschland durchgeführten multizentrischen Phase-II-Studie wurde Docetaxel in einer Dosierung von $100 \mathrm{mg} / \mathrm{m}^{2}$ alle 3 Wochen zur Behandlung von chemotherapeutisch vorbehandelten Patienten mit progredientem metastasierten $\mathrm{M}$ agenkarzinom und messbarer E rkrankung eingesetzt [13]. Von den insgesamt 30 Patienten hatten 23 zuvor eine Kombinationschemotherapie mit 5-Fluorouracil/Folinsäure/ Cisplatin und 7 Patienten eine andere Chemotherapie auf 5-Fluorouracilbasis erhalten. Von 28 auswertbaren Patienten (18\%) sprachen 5 auf die Second-line-Therapie mit D ocetaxel mit einer objektiven R emission an. B ei weiteren 8 Patienten kam es zu einer Tumorstabilisierung, entsprechend einer Tumorwachstumskontrollrate von insgesamt $46 \%$. D amit entsprechen diese E rgebnisse nahezu denen in der First-line-Therapie, so dass davon auszugehen ist, dass zwischen Docetaxel und Cisplatin bzw. 5-Fluorouracil keine klinisch relevante K reuzresistenz besteht. Die Toxizität von Docetaxel entsprach im Wesentlichen der in der First-line-Therapie, 64\% der Patienten zeigten eine N eutropenie $\mathrm{G}$ rad $3 / 4$.

\section{Ausblick und Fazit}

D ie Kombinationstherapie von D ocetaxel und Cisplatin wird derzeit an einer größeren Zahl von Patienten in einer randomisierten Phase-III-Studie evaluiert. Interessant erscheint es aber auch, Docetaxel mit anderen neuen Substanzen, z.B. einem der oralen 5-Fluorouracil-Prodrugs oder dem Topoisomerase-I-H emmer Irinotecan zu kombinieren. In eigenen Invitro-U ntersuchungen konnten wir nachweisen, dass es bei der sequenziellen $\mathrm{G}$ abe von Docetaxel gefolgt von $\mathrm{SN}-38$, dem aktiven $M$ etaboliten von Irinotecan, zu einem deutlichen Synergismus der zytotoxischen Wirkung kommt [11]. A Is möglicher molekularer Mechanismus für diese Wechselwirkung wird eine signifikant höhere Induktion von SN -38 assoziierten proteingebundenen DNA -Strangbrüchen - also Vorstufen von D NA -D oppelstrangbrüchen - nach Vorbehandlung mit D ocetaxel diskutiert. Die Entwicklung eines geeigneten Kombinationsregimes von Docetaxel und Irinotecan scheint daher interessant für zukünftige klinische Prüfungen zu sein.

Aus den bisherigen klinischen $D$ aten zu D ocetaxel beim fortgeschrittenen M agenkarzinom lässt sich zusammenfassen, dass die Substanz bei dieser Erkrankung eine signifikante antitumorale Wirksamkeit besitzt, und zwar in der First-lineTherapie ebenso wie bei Patienten, die zuvor bereits mit einer Chemotherapie auf 5-Fluorouracil- und/oder Cisplatin-Basis behandelt wurden. D ocetaxel besitzt ein tolerables Toxizitätsspektrum, wobei eine ausgeprägte, jedoch kurz dauernde Neutropenie im Vordergrund steht. Die Kombination von Docetaxel mit Cisplatin, aber auch mit anderen Zytostatika wie z.B. I rinotecan, erscheint klinisch sinnvoll. 


\section{Literatur}

1 M eyer HJ, Jahne J, Wilke H, Pichlmayr R: Surgical treatment of gastric cancer: retrospective survey of 1,704 operated cases with special reference to total gastrectomy as the operation of choice. Semin Surg Oncol 1991;7:356-364.

2 Vanhoefer $U$, R ougier P, Wilke H, D ucreux M , L acave A, Van Culsern E, Planker M, G uimaraes D os Santos $J$, Piedbois $P$, Paillot $B$, Bodenstein $H$, Schmoll $H-J$, Bleiberg $H, N$ ördlinger $B$, Couvreur $M-L, B$ aron $B$ Wils JA : Final results of a randomized phase III trial of sequential high-dose methotrexate, fluorouracil, and doxorubicin versus etoposide, leucovorin, and fluorouracil versus infusional fluorouracil and cisplatin in advanced gastric cancer: A trial of the $E$ uropean O rganization for R esearch and Treatment of Cancer Gastrointestinal Tract Cancer Cooperative Group. J Clin O ncol 2000;18:in press

3 Webb A, Cunningham D, Scarffe $J H, H$ arper $P$ Norman $A$, J offe J K, H ughes $M, M$ ansi J, Findlay $M$, $\mathrm{H}$ ill $\mathrm{A}, \mathrm{O}$ ates J, Nicolson $\mathrm{M}, \mathrm{H}$ ickish $\mathrm{T}, \mathrm{O}$ 'B rien $\mathrm{M}$, I veson $T$, Watson $M$, U nderhill $C$, Wardley $A$, M eehan $M: R$ andomized trial comparing epirubicin, cisplatin, and fluorouracil versus fluorouracil, doxorubicin, and methotrexate in advanced esophagogastric cancer. J Clin Oncol 1997;15:261-267.

4 Vanhoefer U, Wilke H, Fink U, K öhne-Wömpner C, Preusser P, Korn W M, Stahl W, H ammick A, K lausem $\mathrm{U}, \mathrm{Schmoll} \mathrm{H-J}$, Schuber S: Weekly 24h-infusion of high dose FU (HD-FU) plus folinic acid (FA) plus biweekly cisplatin (C) (Group A) of HD-FU/FA/C plus epirubicin ( $E$ ) (G roup B) in locally advanced or metastatic gastric cancer. Proc A m Soc Clin O ncol 1995; 14:197a (abstr)

5 Sulkes A , Smyth J, Sessa C, D irix LY, Vermorken J B K aye $\mathrm{S}$, Wanders J, Franklin $\mathrm{H}$, L eB ail N, Verweij J D ocetaxel (TaxotereTM) in advanced gastric cancer: Results of a phase II clinical trial. $\mathrm{Br}$ J Cancer 1994;70:380-383.

6 A ndroulakis N, Kourousis C, Kakolyris S, Sarra E, K albakis K, K alikaki T, Kois S, Vardakis N, Samonis G, G eorgoulias V: M onotherapy with docetaxel and granulocyte colony-stimulating factor in advanced gastric cancer. A nn O ncol 1998;9(suppl 4):258

7 Einzig AI, Neuberg D, Remick SC, Karp DD, $O$ 'D wyer PJ, Stewart JA, B enson A B: Phase II trial of docetaxel (Taxotere) in patients with adenocarci noma of the upper gastrointestinal tract previously untreated with cytotoxic chemotherapy: The E astern Cooperative Oncology Group (ECOG) results of Protocol E 1293. M ed O ncol 1996;13:87-93.

8Taguchi T, Sakata $Y$, Kanamaru R, Kurihara M Suminaga $\mathrm{M}, \mathrm{O}$ ta $\mathrm{J}, \mathrm{H}$ irabayashi $\mathrm{N}$ : Late phase II clinical study of R P56976 (docetaxel) in patients with advanced/recurrent gastric cancer: A Japanese Cooperative Study Group trial (group A). Gan To K agaku R yoho 1998;25:1915-1924.

9 Ridwelski K, G ebauer T, Fahlke J, K röning H, K ettne $E$, M eyer $F$, Lippert $H$ : Combination chemotherapy with docetaxel and cisplatin for locally advanced and metastatic gastric cancer. E ur J Cancer 1999;35 (suppl 4):565.
10 R oth A D, M aibach R, M artinelli G, Fazio N, A apro M S, Pagani O, M orant R, B orner M M , H errmann $R$, $\mathrm{H}$ onegger $\mathrm{HP}, \mathrm{C}$ avalli $\mathrm{F}, \mathrm{A}$ lberto $\mathrm{P}, \mathrm{C}$ astiglione $\mathrm{M}$, Goldhirsch A, on behalf of the Swiss Group for Clinical Cancer R esearch (SA KK):Taxotere-cisplatin (TC) in advanced gastric carcinoma ( $A G C$ ): A n active drug combination. Proc A m Soc Clin Oncol 1998:17:283(abstr 1088)

11 Constenla M , G arcia-A rroyo FR , L orenzo I, Carrete $N$, R ey C:A phase II study of docetaxel (TXT) with weekly high-dose 5-FU \& leucovorin (24-hour infusion) in advanced gastric cancer. Proc A $m$ Soc Clin Oncol 1998;17:1113(abstr).

$12 \mathrm{R}$ oth $\mathrm{A} D$, M aibach $\mathrm{R}, \mathrm{M}$ orant $\mathrm{R}$, Fazio N, Pagani $\mathrm{O}$ De Braud $F$, Borner M M , Stupp $R$, Herrmann $R$ Sessa C, Goldhirsch A: 5FU in protracted continuous IV infusion (5FU piv) can be added to Taxotere-cisplatin (TC) without dose-reduction of TC in advanced gastric carcinoma (A G C). Proc A m Soc Clin Oncol 1999;18:943(abstr).

13 Vanhoefer $\mathrm{U}$, Wilke $\mathrm{H}, \mathrm{H}$ arstrick $\mathrm{A}, \mathrm{A}$ chterrath $\mathrm{W}$, Preusser P, Stahl M, Clemens, M R Thiel E, Flasshove $M$, Fink $U$, Trenn $G$, Seeber S: Phase II study of docetaxel as second line chemotherapy (CT) in metas tatic gastric cancer. Proc A m Soc Clin O ncol 1999 18:1163(abstr)

14 Vanhoefer U, H ilger R A , M üller M R, A chterrath W $\mathrm{H}$ arstrick A, Seeber S: Schedule-dependent cytotoxic interactions of irinotecan (SN-38) and docetaxel in non-small-cell lung cancer. Proc A m A ssoc Cancer R es 2000;41 (abstr). 